\title{
Bilirubin adsorption property of mesoporous silica and amine-grafted mesoporous silica
}

\author{
L. X. Zhang, M. Zhu, L. M. Guo, L. Li and J. L. Shi*
}

Nephritic, hepatic and immune failures would lead to the overload of endogenous toxic molecules (e.g. bilirubin, cholic acid, uric acid, creatinine etc.) in human bodies. It is fatal in most cases and extracorporeal blood purification (ECBP) is powerful first-aid therapy. Adsorbents are key parts of ECBP apparatus. Mesoporous silicas should be promising candidates for these medical adsorbents, but there is no report about this. Herein, pure and amine-grafted mesoporous silicas have been applied to adsorb bilirubin, cholic acid, uric acid, creatinine and phenobarbital for the first time. These mesoporous materials show high adsorption capacities for bilirubin and uric acid in phosphate buffer solution (PBS). Effects of pore sizes, amine-modification, temperature and ionic strength on their bilirubin adsorption capacities have been studied in detail.

Keywords: Mesoporous silica; Bilirubin adsorption; Amine-grafted

Citation: L. X. Zhang, M. Zhu, L. M. Guo, L. Li and J. L. Shi, "Bilirubin adsorption property of mesoporous silica and amine-grafted mesoporous silica”, Nano-Micro Lett. 1, 14-18 (2009). doi: 10.5101/nml.v1i1.p14-18

Generally, there are exogenous and endogenous poisons overloading in human body [1]. Acute medicament (e.g. soporific) toxicosis is the most common exogenous case. Nephritic, hepatic and immune failures lead to overload of endogenous toxic molecules (e.g. bilirubin, cholic acid, uric acid, creatinine etc.) and pull patients to death's door. Extracorporeal blood purification (ECBP), including hemodialysis, hemofiltration, hemoperfusion and plasma exchange, is powerful first-aid therapy to remove toxins from human hematic system [2]. Adsorbents are the most important parts of ECBP apparatus. Active carbon, chitosan and resins have been applied in ECBP [3-6]. However, development of medical adsorbents with high adsorption capacity and excellent blood compatibility is still a great challenge. Mesoporous silicas, which have high surface area, uniform pore size and abundant $\mathrm{Si}-\mathrm{OH}$ groups, have been used as adsorbents for heavy metal ions [7-10] and organic pollutants [10] and toxic gas [10], etc. Recently, they have also been applied to medicine storage and targeting therapy [7, 11, 12]. Mesoporous silicas should also be promising candidates for medical adsorbents of ECBP to remove the above-mentioned toxins from human hematic system. However, there are few reports about this. Herein this article, pure and amine-grafted mesoporous silicas have been prepared and applied to adsorb bilirubin, cholic acid, uric acid, creatinine, etc. Effects of pore size, amine-grafting, temperature and ionic strength on the bilirubin adsorption capacities of these materials have been studied in detail.

\section{EXPERIMENTAL}

A. Materials. Bilirubin ( $99 \%)$ was supplied from Alfa Aesar Chemical Company. The molecular structure of bilirubin has been shown in Fig. 1. Cholic acid (>99\%) was obtained from Fluka Chemical Company. Uric acid (>99\%), creatinine ( $>99 \%$ ), tetraethyl orthosilicate (TEOS, $>99 \%$ ), were got from SCRC (Sinopharm Chemical Reagent Company, China). All reagents are used as received. 
<smiles>C=CC1=C(C)/C(=C/c2[nH]c(Cc3[nH]c(/C=C4\NC(=O)C(C=C)=C4C)c(CCC(=O)O)c3C)c(CCC(=O)O)c2C)NC1=O</smiles>

FIG. 1. The molecular structure of bilirubin.

\section{B. Preparation and amine-modification of mesoporous}

silica SBA-15. Mesoporous silica SBA-15 was synthesized using a triblock copolymer surfactant in acid media according to a published procedure [13]. In a typical synthesis, $4 \mathrm{~g}$ of block copolymer surfactant EO20PO70EO20 (Pluronic P123, BASF) was dissolved in $30 \mathrm{~g}$ of distilled water and $120 \mathrm{ml}$ of 2 $\mathrm{M} \mathrm{HCl}$ with stirring, followed by addition of $8.5 \mathrm{~g}$ of TEOS at $35-40^{\circ} \mathrm{C}$. This gel was continuously stirred for $24 \mathrm{~h}$ and then crystallized in a glass bottle at $100{ }^{\circ} \mathrm{C}$ for $48 \mathrm{~h}$. After crystallization, the solid product was filtered, washed with distilled water, and dried in air at $100^{\circ} \mathrm{C}$. The material was calcined in air at $530^{\circ} \mathrm{C}$ for $6 \mathrm{~h}$ to remove the surfactant template and obtain a white parent powder (SBA-15). For preparation of larger pore SBA-15, 2 g of P123 was dissolved in $75 \mathrm{ml} 1 \mathrm{M} \mathrm{HCl}$ with stirring, followed by addition of $1.38 \mathrm{ml}$ $0.5 \mathrm{M} \mathrm{NH}_{4} \mathrm{~F}$ and $8.5 \mathrm{~g}$ of TEOS at $35-40^{\circ} \mathrm{C}$. This gel was continuously stirred for $24 \mathrm{~h}$ and then crystallized in a sealed glass bottle at $110^{\circ} \mathrm{C}$ for $48 \mathrm{~h}$. FSBA-15 was then obtained after filtration and calcination.

Multi-amine grafted SBA-15 and FSBA-15 was prepared according to published procedures $[14,15]$. Functional groups (amine, diamine and triamine groups in this case) were introduced to the pore surfaces of mesoporous silica by refluxing the mixture of $1 \mathrm{~g}$ of the calcined SBA-15 or FSBA15 and $5 \mathrm{ml}$ of $\mathrm{\gamma}$-aminopropyltriethoxysilane (APTS), N-[3(trimethoxysilyl)-propylethylene] diamine (TPED), trimethoxysilyl propyl diethylenetriamine (TPDT) in $100 \mathrm{ml}$ of dry toluene under nitrogen atmosphere for at least $12 \mathrm{~h}$. The resulting hybrid materials (denoted as $1 \mathrm{~N}-\mathrm{SBA}-15,2 \mathrm{~N}-\mathrm{SBA}-15$, $3 \mathrm{~N}-\mathrm{SBA}-15,2 \mathrm{~N}-\mathrm{FSBA}-15$ and so on) were filtered out using toluene thoroughly and dried in vacuum at $70-80^{\circ} \mathrm{C}$.

C. Preparation of mesoporous silica KIT-6. The mesoporous silica with cubic Ia3d symmetry (designated as KIT-6) was prepared according to the published procedure using tri-block copolymer Pluronic P123 as template by adding of n-butanol in an acidic aqueous solution [16]. Typically, $6 \mathrm{~g}$ of surfactant P123 and $6 \mathrm{~g}$ of n-butanol were dissolved in the solution of $217 \mathrm{~g}$ of distilled water and $11.4 \mathrm{~g}$ of concentrated $\mathrm{HCl}$ (37 wt \%). To this homogeneous solution, $12.9 \mathrm{~g}$ of TEOS was added under stirring at $37^{\circ} \mathrm{C}$ and continuously stirred for $24 \mathrm{~h}$. After that, the mixture was transferred to Teflon-lined autoclaves and heated at $100^{\circ} \mathrm{C}$ for $24 \mathrm{~h}$. The solid product was filtered, washed with distilled water and calcined at $550^{\circ} \mathrm{C}$ for $5 \mathrm{~h}$ to get KIT-6.

D. Preparation of mesoporous silica MCM-41. Pure silica MCM-41 samples were prepared by the hydrolysis of cetyl-trimethyamonium bromide $(\mathrm{CTAB})$ and TEOS in basic solution according to the published procedure. The final composition of the gel is 1.0 CTAB: 7.5 TEOS: $1.8 \mathrm{NaOH}: 500$ $\mathrm{H}_{2} \mathrm{O}$ (molar ratio). The gel was hydrothermally treated at $110^{\circ} \mathrm{C}$ for $60 \mathrm{~h}$ and calcined at $550^{\circ} \mathrm{C}$ for $5 \mathrm{~h}$ to obtain MCM-41 $[17,18]$

E. Preparation of toxins solutions. Bilirubin stock solution was prepared by dissolving $250 \mathrm{mg}$ of bilirubin in 10 $\mathrm{ml} 0.06 \mathrm{M} \mathrm{NaOH}$ solution and then diluting this solution to 250 $\mathrm{ml}$ by phosphate buffer solution (PBS, $\mathrm{pH}=7.4$ ). Bilirubin solutions of lower concentrations were obtained by further dilution using PBS. All solutions were stored in refrigerator in dark.

Uric acid solution was prepared by dissolving $500 \mathrm{mg}$ of uric acid in $250 \mathrm{ml}$ of $0.02 \mathrm{M} \mathrm{NaOH}$ solution. A $500 \mathrm{mg}$ of creatinine was dissolved in $100 \mathrm{ml} \mathrm{H}_{2} \mathrm{O}$ to prepare creatinine solution. To prepare phenobarbital solution, five phenobarbital tablets (30mg per tablet, pet using only) were ground and

Table 1. Structure parameters of mesoporous silicas and their adsorption capacities for bilirubin.

\begin{tabular}{ccccc}
\hline Samples & $\begin{array}{c}\text { BET surface area } \\
\left(\mathrm{m}^{2} / \mathrm{g}\right)\end{array}$ & $\begin{array}{c}\text { BJH desorption } \\
\text { pore diameter } \\
(\mathrm{nm})\end{array}$ & $\begin{array}{c}\text { BJH desorption } \\
\text { pore volume } \\
\left(\mathrm{cm}^{3} / \mathrm{g}\right)\end{array}$ & $\begin{array}{c}\text { Adsorption capacities for } \\
\text { bilirubin } \\
(\mathrm{mg} / \mathrm{g})\end{array}$ \\
\hline MCM-41 & 708 & 2.6 & 0.60 & 63 \\
KIT-6 & 684 & 5.8 & 0.79 & 52 \\
SBA-15 & 775 & 6.8 & 1.20 & 37 \\
FSBA-15 & 534 & 8.8 & 1.28 & 37 \\
1N-SBA-15 & 373 & 6.0 & 0.76 & 51 \\
2N-SBA-15 & 336 & 6.1 & 0.74 & 47 \\
3N-SBA-15 & 256 & 6.1 & 0.57 & 42 \\
2N-FSBA-15 & 266 & 7.9 & 0.71 & 51 \\
\hline
\end{tabular}


dissolved in $100 \mathrm{ml} \mathrm{H}_{2} \mathrm{O}$. Cholic acid was prepared by dissolving $50 \mathrm{mg}$ of Cholic acid in $6 \mathrm{ml} 0.05 \mathrm{M} \mathrm{NaOH}$ solution and then diluting this solution to $100 \mathrm{ml}$ using PBS.

\section{CHARACTERIZATION}

Concentrations of all toxins are characterized using UV-Vis absorbance, which was measured using Shimadzu UV-3101PC spectroscope. $\mathrm{N}_{2}$ adsorption-desorption isotherms were carried out on Micromeritics Tristar 3000 analyzer at $77 \mathrm{~K}$ under a continuous adsorption condition. BET (BrunauerEmmet-Teller) and BJH (Barrett-Joyner-Halenda) analyses were used to determine the surface area, pore size and volume.

A. Effect of pore sizes of mesoporous silicas on bilirubin adsorption. As shown in Tab.1, pure mesoporous silicas MCM-41, KIT-6, SBA-15 and FSBA-15 with pore diameters from 2.6 to $8.8 \mathrm{~nm}$ have adsorbed $63,52,37$ and 37 $\mathrm{mg} / \mathrm{g}$ bilirubin, respectively. Small pore diameters are favorable to get high adsorption capacity. The samples SBA-15 and FSBA-15 have the same adsorption capacities, which could be attributed to their microporous structure in pore wall frameworks. However, surface areas of these pure mesoporous silicas have little influence on their adsorption capacities for bilirubin.

\section{B. Effect of amine-modification of mesopore surface on} bilirubin adsorption. For comparison, amine-grafted mesoporous silicas SBA-15 have also been synthesized and applied to adsorb bilirubin. 1N-SBA-15, 2N-SBA-15 and 3N-SBA-15 have similar pore diameters (about $6 \mathrm{~nm}$ ). Large pore volumes and high surface areas have lead to slightly increase of their adsorption capacities from 42 to $51 \mathrm{mg} / \mathrm{g}$. More amine groups have not improved their bilirubin adsorption capacities. In order to study on the influence of pore diameter of amine-grafted mesoporous silicas on their bilirubin adsorption capacities, 2N-FSBA-15 has been also given as an

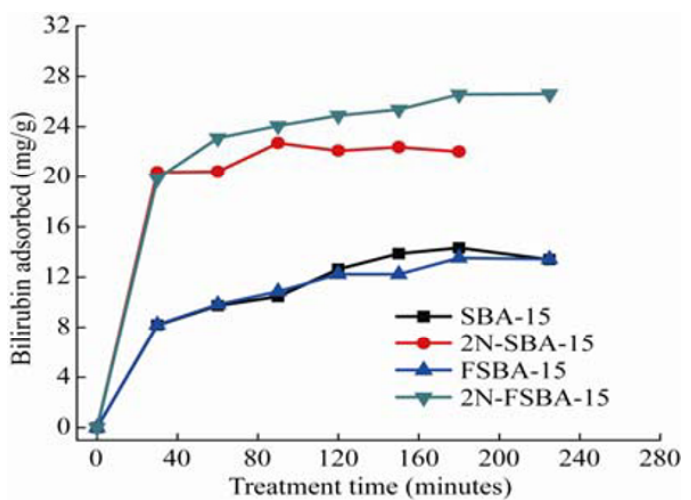

FIG. 2. Influence of the adsorption time on the adsorption capacities of the mesoporous silica adsorbents for bilirubin. Initial concentration of bilirubin: 300 $\mathrm{mg} / \mathrm{L}, 10 \mathrm{ml}$; mesoporous adsorbent: $10 \mathrm{mg}$; temperature: $310 \mathrm{~K}$.

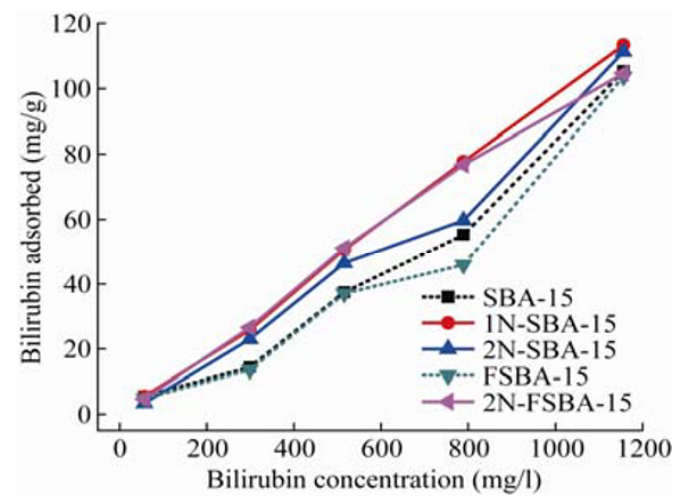

FIG. 3. Influence of initial concentrations of bilirubin on the adsorption capacities of mesoporous silica adsorbents for bilirubin. Adsorption time: $3 \mathrm{~h}$; temperature: $310 \mathrm{~K}$; bilirubin solution: 10ml; mesoporous silica adsorbents: $10 \mathrm{mg}$.

example. 2N-FSBA-15 with pore diameter $7.9 \mathrm{~nm}$ has adsorbed $51 \mathrm{mg} / \mathrm{g}$ bilirubin. This adsorption capacity is higher than that of 2N-SBA-15 sample, although 2N-FSBA-15 has smaller surface area and pore volume than $2 \mathrm{~N}-\mathrm{SBA}-15$. This indicates that large pore diameters rather than $\mathrm{N}$ numbers are beneficial to improve the adsorption capacities of amine-grafted samples. It can be attributed to the high flexibility of amine group chains in large pore channels.

For SBA-15 and FSBA-15, as shown in Table 1, the surface areas, pore diameters and volumes all decrease after the introduction of amine groups (2N-SBA-15 and 2N-FSBA-15). However, all amine-grafted mesoporous silicas show higher adsorption capacities than their pure parent mesoporous silicas. The same results can also be obtained from Fig. 2. Their bilirubin adsorption capacities of 2N-SBA-15 and 2N-FSBA-15 are higher than those of SBA-15 and FSBA-15, which is independent of the treatment time. As reported in literatures before [19], introductions of hydrophobic groups in adsorbents can effectively improve their adsorption capacities for

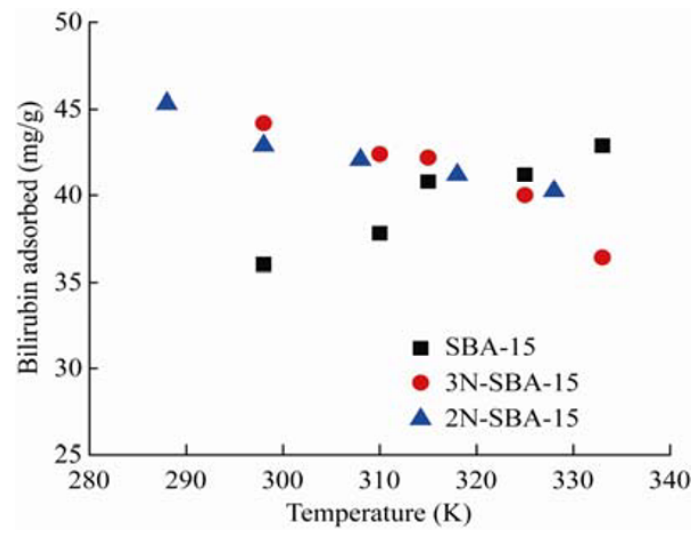

FIG. 4. Influence of temperature on the adsorption capacities of mesoporous silica adsorbents for bilirubin. Initial concentration of bilirubin: $500 \mathrm{mg} / 1,10 \mathrm{ml}$; adsorption time: $3 \mathrm{~h}$; mesoporous silica adsorbents: $10 \mathrm{mg}$. 
Table 2. Adsorption properties of mesoporous silicas for cholic acid, uric acid, creatinine and phenobarbital.

\begin{tabular}{cccccc}
\hline & \multicolumn{5}{c}{ Adsorption capacities (mg/g) } \\
\cline { 2 - 5 } & SBA-15 & MCM-41 & 1N-SBA-15 & 2N-SBA-15 & 3N-SBA-15 \\
\hline Cholic acid & 52 & 70 & 0 & 0 & 0 \\
Uric acid & 118 & 108 & 74 & 0 & 0 \\
creatinine & 0 & 0 & 0 & 0 & 0 \\
phenobarbital & 0 & 0 & 0 & 0 \\
\hline
\end{tabular}

Initial concentration of Cholic acid: $1500 \mathrm{mg} / 1,10 \mathrm{ml}$; temperature: $293 \mathrm{~K}$; mesoporous adsorbents: $10 \mathrm{mg}$; adsorption time: $6 \mathrm{~h}$.

Initial concentration of Uric acid: $2000 \mathrm{mg} / 1,10 \mathrm{ml}$; temperature: $293 \mathrm{~K}$; mesoporous adsorbents: $10 \mathrm{mg}$; adsorption time: $6 \mathrm{~h}$.

liposoluble bilirubin. Herein the grafting of amine-containing silane chains onto the pore surface makes these mesoporous adsorbents more hydrophobic and leads to increasing adsorption capacities.

\section{Effect of initial concentrations of bilirubin. Figure 3} shows the influence of initial concentrations of bilirubin on the adsorption capacities of pure and amine-grafted mesoporous silicas. Their adsorption capacities all increase with the increase of initial concentrations of bilirubin. Amine-grafted mesoporous silicas show higher adsorption capacities than pure mesoporous silicas, which is unreliable to the initial concentrations of bilirubin. 1N-SBA-15 and 2N-FSBA-15 adsorbed similar amount of bilirubin because of the similar flexibility of amine group chains in their pore channels. 2N-SBA-15 show less bilirubin capacity than $1 \mathrm{~N}-\mathrm{SBA}-15$ and $2 \mathrm{~N}-\mathrm{FSBA}-15$, which can be ascribed to its small pore size and less flexibility of amine-containing organic chains.

\section{Effect of temperature on bilirubin adsorption.} Experiments on the bilirubin adsorption under different temperatures have been conducted and the results are shown in Fig. 4. The bilirubin adsorption capacities of SBA-15 increase while those of amine-grafted SBA-15 decrease when the experiment temperatures become higher. For pure mesoporous

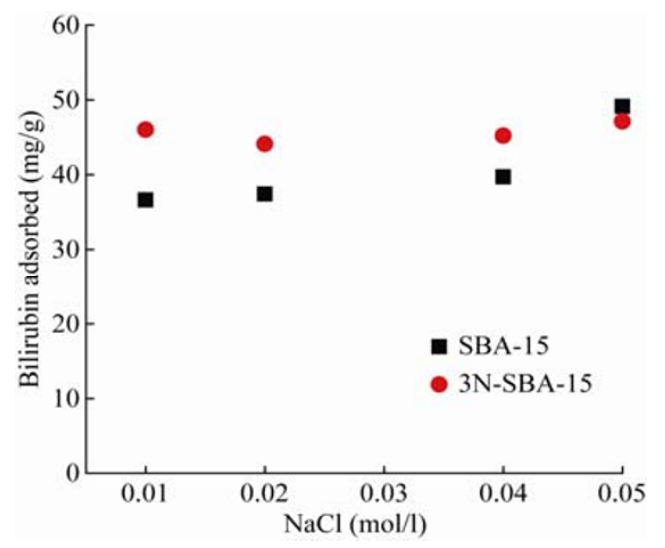

FIG. 5. Effect of ionic strength on the adsorption capacities of mesoporous silica adsorbents for bilirubin. Initial concentration of bilirubin: $500 \mathrm{mg} / 1,10 \mathrm{ml}$; adsorption time: $3 \mathrm{~h}$; mesoporous silica adsorbents: $10 \mathrm{mg}$; temperature: $298 \mathrm{~K}$. silicas, hydrogen bonds between Si-OH groups of mesoporous silicas and the - $\mathrm{COOH}$, -NH- groups of bilirubin can be formed in PBS. With the increase of temperature, diffusion of bilirubin molecules into the pore channels of mesoporous adsorbents have greatly improved and accordingly the amount of hydrogen bonds also increased, thus their adsorption capacities increase. As for amine-grafted mesoporous silicas, adsorption mainly bases on the electrostatic interaction or the ionic bonds between $-\mathrm{NH}_{2} /-\mathrm{NH}$-groups of mesoporous adsorbents and - $\mathrm{COOH}$ groups of bilirubin molecules. These charge effects have been greatly weakened under high temperature because the diffusion of bilirubin in the pore channels of adsorbents is accelerated.

\section{E. Effect of ionic strength on bilirubin adsorption.} Figure 5 shows the influence of ionic strength $(\mathrm{NaCl}$ concentrations) of the PBS solution on their adsorption capacities of pure and amine-grafted mesoporous silicas. With the increase of the concentration of $\mathrm{NaCl}$, the ionic atmosphere around - $\mathrm{COO}^{-}$groups of bilirubin increases. On the one hand, the formation of the ionic atmosphere makes the bilirubin molecules get more hydrophilic. Therefore, bilirubin adsorption capacities for pure mesoporous silica SBA-15 increase when the ionic strength of the solutions increases. On the other hand, the ionic atmosphere around $-\mathrm{COO}^{-}$groups of bilirubin also leads to obvious steric hindrance. At the same time, the ionic atmosphere around amine groups on the pore surface of amine-grafted mesoporous silicas also causes obvious steric hindrance. These steric hindrance effects make adsorption capacities of amine-grafted mesoporous silicas decrease. Meanwhile, the improved hydrophilic bilirubin is favorable to get high adsorption capacity. The banlance between these two facts makes the bililirubin adsorption capacities of 3N-SBA-15 keep between $40-50 \mathrm{mg} / \mathrm{g}$ when the ionic strength has been changed.

F. Adsorption for other toxins. Pure and amine-grafted mesoporous silicas have also been applied to adsorb other toxins, such as cholic acid, uric acid, creatinine. As shown in Table 2, pure mesoporous silicas also show high adsoprtion 
capacity for cholic acid. However, amine-grafted mesoporous silicas do not adsorb cholic acid at all. Both pure mesoporous silicas and amine-grafted mesoporous silicas show high adsorption capacities for uric acid. Neither pure mesoporous silicas nor amine-grafted mesoporous silicas have adsorption capacities for creatinine and phenobarbital. Further studies on the adsorption of these toxins on mesoporous adsorbents are still in progress.

\section{CONCLUSION}

Both pure and amine-grafted mesoporous silicas show high adsorption capacities for bilirubin and uric acid. Pure mesoporous silicas with smaller pore size show higher adsorption capacities for bilirubin. For amine-grafted samples, larger pore diameters, rather than $\mathrm{N}$ numbers, are beneficial for the enhancement of their adsorption capacities, which can be attributed to the high flexibility of amine-containing silane chains in large pore channels. Amine modification of mesoporous silicas can increase their adsorption capacities for bilirubin because the grafting of amine-containing silane chains onto the pore surface makes these mesoporous adsorbents more hydrophobic and then leads to increased adsorption capacities of hydrophobic bilirubin. Increase in temperature leads to the enhanced bilirubin adsorption capacities of pure mesoporous silica because diffusion of bilirubin molecules into the pore channels have greatly improved and the amount of hydrogen bonds between $\mathrm{Si}-\mathrm{OH}$ and $-\mathrm{COOH} /-\mathrm{NH}-$ also increases. However, the bilirubin adsorption capacities of amine-grafted samples decrease at increased temperature because of the faster bilirubin diffusion in the pore channels of adsorbents and the weaker ionic bonds between the -NH2/-NH- of mesoporous adsorbents and $-\mathrm{COOH}$ of bilirubin at higher temperature. With the increase of ionic strength, the bilirubin adsorption capacities of pure mesoporous silicas increase due to the formation of the ionic atmosphere which makes the bilirubin molecules more hydrophilic. The bilirubin adsorption capacities of aminegrafted mesoporous silicas change little resulted from the counteraction from the steric hindrance effect of the ionic atmosphere. Pure mesoporous silicas also have affinity to cholic acid. Neither pure mesoporous silicas nor amine-grafted mesoporous silicas can adsorb creatinine and phenobarbital.

The authors gratefully acknowledge the financial supports from the National Science Foundation of China (Grant No. 50702072) and the Shanghai "Phosphor" Science Foundation, China (Grant No. 08QA14074).
Received 16 September 2009; accepted 2 October 2009; published online 15 October 2009.

\section{References}

1. R. Guo, L. Y. Jia, H. Q. Feng and C. Y. Zou, Chin. J. Blood Purificat. 3, 43 (2004).

2. A. E. Briglia, Semin. Dialysis. 18, 380 (2005).

3. J. D. Zhang and Z. Yuan, Chin. Polym. Bull. 4, 25 (2006).

4. Z. Yang, S. Si and Y. Fung, Thin Solid Films 515, 3344 (2007). doi:10.1016/j.tsf.2006.09.018

5. M. C. Annesini, C. D. Carlo, V. Piemote and L. Turchetti, Biochem. Eng. J. 40, 205 (2008).

6. Y. Yang, Y. T. Wang and W. A. Zhang, J. Appl. Polym. Sci. 99, 1264 (2006). doi:10.1002/app.22652

7. J. L. Shi, Z. L. Hua and L. X. Zhang, J. Mater. Chem. 14, 795 (2004). doi:10.1039/b315861f

8. X. D. Feng, G. E. Fryxell, L. Q. Wang, A. Y. Kim, J. Liu and K. M. Kemner, Science 276, 923 (1997). doi:10.1126/ science. 276.5314 .923

9. L. X. Zhang, W. H. Zhang, J. L. Shi, Z. L. Hua, Y. S. Li and J. N. Yan, Chem. Commun. 2, 210 (2003). doi:10.1039/b210457a

10. G. E. Fryxell and G. Z. Cao, Imperial College Press (2007).

11. W. R. Zhao, J. L. Gu, L. X. Zhang, H. R. Chen and J. L. Shi, J. Am. Chem. Soc. 127, 8916 (2005). doi:10.1021/ ja051113r

12. Y. F. Zhu, J. L. Shi, W. H. Shen, X. P. Dong, J. W. Feng, M. L. Ruan and Y. S. Li, Angew. Chem. Int. Ed. 44, 5083 (2005). doi:10.1002/anie.200501500

13. D. Y. Zhao, Q. S. Huo, J. Feng, B. F. Chmelka and G. D. Stucky, J. Am. Chem. Soc. 120, 6024 (1998). doi:10.1021/ ja974025i

14. H. Yoshitake, T. Yokoi and T. Tatsumi, Chem. Mater. 15, 1713 (2003). doi:10.1021/cm0218007

15. L. X. Zhang, C. C. Yu, W. R. Zhao, Z. L. Hua, H. R. Chen, L. Li and J. L. Shi, J. Non-Cryst. Solids 353, 4055 (2007). doi:10.1016/j.jnoncrysol.2007.06.018

16. F. Kleitz, S. H. Choi and R. Ryoo, Chem. Commun. 2136 (2003). doi:10.1039/b306504a

17. C. T. Kresge, M. E. Eonowicz, W. J. Roth, J. C. Vartuli and J. S. Beck, Nature 359, 710 (1992). doi:10.1038/ $\underline{359710 \mathrm{a} 0}$

18. W. H. Zhang, J. L. Shi and H. R. Chen, Chem. Mater. 13, 648 (2001). doi:10.1021/cm000621r

19. Y. H. Yu and B. L. He, React. Funct. Polym. 31, 195 (1996). doi:10.1016/1381-5148(96)00051-X 